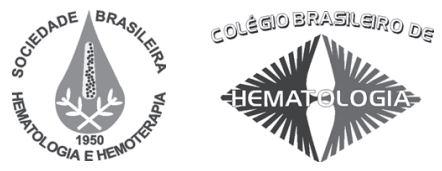

Review / Revisão

\title{
Is there a role for allogeneic haemopoietic stem cell transplants (HSCT) in patients with Hodgkin's disease?
}

Há um papel para o transplante alogênico em paciente com doença de Hodgkin?

Andrea Bacigalupo

\section{Introduction}

Allogeneic HSCT was first investigated in selected patients with advanced HD, and proved that long-term survival improved for some patients who failed many other lines of therapy: patients were prepared with a conventional myeloablative conditioning regimen (MA).

\section{Myeloablative conditioning regimen}

There are 4 studies reporting myeloablative conditioning in preparation for an allogeneic HSCT; the International Bone Marrow Transplant Registry (IBMTR), the European Group for Blood and Marrow Transplantation (EBMT), the Johns Hopkins (JHOC) and the Seattle group (FHCRC) (Table 1).

The total number of patients reported is 373 , with an early transplant-related mortality (TRM) rate of $25 \%$, but an overall cumulative incidence of $52 \%$. The average overall survival was $44 \%$ and progression free survival (PFS) was $20 \%$; the incidence of relapse was $57 \%$ despite of the use of high dose chemo/radiotherapy. Although overall survival was above $44 \%$, the high TRM convinced most hematologists that allogeneic HSCT was not a real option for patients with $\mathrm{HD}$, and the number of transplants per year remained low.

\section{Reduced intensity conditioning (RIC)}

The development of reduced intensity conditioning regimens (RIC) in the late nineties appeared as an opportunity to again test allogeneic HSCT in HD. Several studies have been published on the outcomes of HD patients receiving allogeneic HSCT after a RIC regimen. The early (9\%) and overall (24\%) TRM is significantly reduced compared to MA transplants (25\% and 52\%, respectively) and there was also a 10\% improvement in PFS and OS. Relapse remains a problem (Table 2).

The results we have outlined are representative of the average patient with HD who is referred for an allogeneic HSCT: these are patients who have all failed first line therapy, either because of resistance or early relapse, and most of them have failed an autologous transplant; a few may have been referred to HSCT because they were poor mobilizers,

Divisione di Ematologia e Trapianto di Midollo Osseo, Ospedale San Martino, Genova, Italy.

Correspondence: Andrea Bacigalupo

Divisione di Ematologia e Trapianto di Midollo Osseo,

Ospedale San Martino, Largo Rosanna Benzi, 10

16132 - Genova, Italy

Phone: +329010355469 - Fax.: +39010355583

E-mail:andrea.bacigalupo@h.sanmartino.it 
and could not complete the autologous transplant program. However, there is a significant degree of variability in performance status, chemosensitivity of the disease and outcome after allogeneic HSCT.

Recently the EBMT analyzed 285 HD patients undergoing RIC transplants with the aim of identifying predictors of outcome (Robinson et al. 2008) and showed that progression free survival (PFS) was predicted by performance status at transplant (Karnofsky score > 80) and chemosensitive disease: patients with neither these 2 factors had a PFS of $42 \%$, compared to $8 \%$ for patients with one or more of these 2 predictors. Transplant mortality was also predicted by the same two variables and by older age ( $>45$ years). Donor lymphocyte infusions (DLI) were given to 64 of the 285 patients with a clinical response being reported in $27 \%$ of cases.

Very recently the Seattle team compared transplants from HLA identical sibling $(\mathrm{n}=34)$, unrelated $(\mathrm{n}=24)$ or family haploidentical $(\mathrm{n}=21)$ donors: $\mathrm{PFS}$ was $20 \%, 27 \%$ and $60 \%$, respectively suggesting haploidentical transplants are a new option in HD patients (Seattle ASH 2007).

\section{Allogeneic transplants vs. chemotherapy in patients relapsing after an autograft}

A recent paper (Castagna 2009) looked at HD patients relapsing after an autograft: 24 patients had a donor (related or unrelated) and were transplanted, whereas 20 patients found no donor and received further chemotherapy. The 2year overall survival was $71 \%$ for the transplant group compared to $50 \%$ for the chemo group ( $\mathrm{p}=0.03$ ).

\section{Conclusions}

In conclusion allogeneic HSCT is feasible in patients with $\mathrm{HD}$ and may prolong survival in patients relapsing after an autograft. Results of HLA identical sibling transplants and unrelated donor transplants are comparable. RIC transplants are associated with significantly lower transplant mortality, when compared to myeloablative transplants. However progression free survival is highly dependent on chemoresistance and performance status. Best results are seen in patients with a Karnofsky score greater than 80 and a chemosensitive disease, which raises the issue of considering an allogeneic HSCT before the end stage of the disease.

\section{Resumo}

O transplante alogênico de célula-tronco hematopoética foi inicialmente explorado em pacientes selecionados com DH avançada e provou, em alguns pacientes que falharam a várias linhas terapêticas, promover longa sobrevida. Existem quatro estudos utilizando regime mieloablativo de condicionamento. Apesar da

\begin{tabular}{|c|c|c|c|c|c|c|c|}
\hline Study & n.pts & $\begin{array}{l}\text { TRM } \\
\text { Early }\end{array}$ & $\begin{array}{c}\text { TRM } \\
\text { cumulative }\end{array}$ & $e^{\text {PFS }}$ & OS & Relapse & e Reference \\
\hline IBMTR & 100 & $13 \%$ & $61 \%$ & $15 \%$ & $6 \quad 65 \%$ & $65 \%$ & $\begin{array}{c}\text { Gaiewski } \\
\text { JCO, } 1996\end{array}$ \\
\hline EBMT & 167 & $31 \%$ & $48 \%$ & $16 \%$ & $6 \quad 61 \%$ & $61 \%$ & $\begin{array}{c}\text { Penikef } \\
\text { BMT, } 2003\end{array}$ \\
\hline $\mathrm{JHOC}$ & 53 & $32 \%$ & $43 \%$ & $26 \%$ & $630 \%$ & $53 \%$ & $\begin{array}{l}\text { Akpek } \\
\text { JCO, } 2001\end{array}$ \\
\hline FHCRC & 53 & & $58 \%$ & $22 \%$ & $6 \quad 21 \%$ & $48 \%$ & $\begin{array}{l}\text { Anderson } \\
\text { JCO, } 1993\end{array}$ \\
\hline Total & 373 & $25 \%$ & $52 \%$ & $20 \%$ & $44 \%$ & $57 \%$ & \\
\hline \multicolumn{8}{|c|}{ Table 2. Reduced intensity conditioning (RIC) } \\
\hline Study & n.pts & $\begin{array}{l}\text { TRM } \\
\text { Early }\end{array}$ & $\begin{array}{c}\text { TRM } \\
\text { cumulative }\end{array}$ & PFS & os & Relapse & Reference \\
\hline EBMT & 311 & $17 \%$ & $27 \%$ & $26 \%$ & $46 \%$ & $64 \%$ & $\begin{array}{c}\text { Robinson } \\
\text { Blood, } 2004\end{array}$ \\
\hline UKCG & 49 & $4 \%$ & $16 \%$ & $39 \%$ & $55 \%$ & $43 \% \backslash$ & $\begin{array}{c}\text { Peggs } \\
\text { Lancet, } 2005\end{array}$ \\
\hline MDAH & 58 & $2 \%$ & $15 \%$ & $32 \%$ & $64 \%$ & $55 \%$ & $\begin{array}{c}\text { Anderlini } \\
\text { BBMT, } 2008\end{array}$ \\
\hline FHCRC & 27 & $11 \%$ & $39 \%$ & $18 \%$ & $51 \%$ & $47 \%$ & $\begin{array}{c}\text { Burroughs } \\
\text { BBMT, } 2004\end{array}$ \\
\hline SPCP & 40 & $12 \%$ & $25 \%$ & $32 \%$ & $48 \%$ & & $\begin{array}{c}\text { Alvarez } \\
\text { BBMT, 2006 }\end{array}$ \\
\hline GITMO & 32 & & & & $32 \%$ & & $\begin{array}{c}\text { Corradini } \\
\text { JCO, } 2007\end{array}$ \\
\hline Total & 485 & $9 \%$ & $24 \%$ & $29 \%$ & $53 \%$ & $52 \%$ & \\
\hline
\end{tabular}

sobrevida global de 44\%, as altas taxas de mortalidade relacionadas ao procedimento convenceram a maioria dos hematologistas que esta não é uma opção real para pacientes com $\mathrm{DH}$. O desenvolvimento do transplante de intensidade reduzida (RIC) nos anos 90 mostrou ser esta uma área de oportunidade para o transplante alogênico em DH. RIC foi associado com significante redução das taxas de mortalidade e comparável aos ablativos em termos de sobrevida global. Entretanto, a PFS é altamente dependente da quimiorresistência e do "performance status". Rev. Bras. Hematol. Hemoter. 2009; 31 (Supl. 2):7-8.

Palavras-chave: Doença de Hodgkin; transplante alogênico de célula-tronco.

O tema apresentado foi proposto pela Organização do $8^{\circ}$ Simpósio da Associação Ítalo-Brasileira de Hematologia, realizado durante o XIII Congresso de Transplante de Medula Óssea.

Publicado após concordância do editor.

Conflito de interesse: sem conflito de interesse

Recebido: 16/07/2009

Aceito: 17/07/2009 